\title{
$\alpha$-lipoic acid protects against cerebral ischemia/reperfusion-induced injury in rats
}

\author{
HOULIANG DENG ${ }^{1 *}$, XIALIN ZUO ${ }^{2 *}$, JINGJING ZHANG ${ }^{1}$, XIAOXIA LIU ${ }^{1}$, \\ LI LIU $^{1}$, QIAN XU ${ }^{1}$, ZHUOMIN WU ${ }^{1}$ and AIMIN JI ${ }^{1}$ \\ ${ }^{1}$ Center for Drug Research and Development, Zhujiang Hospital, Southern Medical University, Guangzhou, \\ Guangdong 510282; ${ }^{2}$ Institute of Neurosciences and The Second Affiliated Hospital of Guangzhou Medical University, \\ Guangzhou, Guangdong 510260, P.R. China
}

Received December 16, 2013; Accepted October 1, 2014

DOI: $10.3892 / \mathrm{mmr} .2015 .3170$

\begin{abstract}
It is well established that the brain is sensitive to ischemia/reperfusion (I/R)-induced injury. $\alpha$-lipoic acid (LA), a free radical scavenger and antioxidant, has a neuroprotective effect against cerebral I/R-induced injury, however, the underlying mechanisms remain to be elucidated. Therefore, the present study was undertaken to evaluate whether LA was able to protect against cerebral I/R-induced injury and to examine the potential mechanisms. The neuroprotective effects of LA were investigated in a rat model of transient focal ischemia induced by middle cerebral artery occlusion (MCAO) followed by reperfusion. Adult male Sprague-Dawley rats were randomly assigned into the sham, cerebral I/R injury model and model plus LA groups. Cerebral I/R injury was induced by $90 \mathrm{~min}$ MCAO followed by reperfusion for $24 \mathrm{~h}$. Cerebral infarct size was detected by 2,3,5-triphenyltetrazolium chloride staining. Neurological deficit score (NDS), brain water content and oxidative parameters, including malondialdehyde (MDA), nitric oxide (NO), total antioxidant capacity (T-AOC) and superoxide dismutase (SOD) were measured. The expression of cleaved caspase-3, brain-derived neurotrophic factor (BDNF), phosphatidylinositol-4,5-bisphosphate 3-kinase (PI3K), p-Akt and phosphorylated extracellular signal-regulated kinase 1/2 (p-ERK1/2) were also analyzed using western blotting. The present study demonstrated that pretreatment with LA significantly decreased the infarction size, brain water content and improved NDS. LA reversed the levels
\end{abstract}

Correspondence to: Professor Aimin Ji, Center for Drug Research and Development, Zhujiang Hospital, Southern Medical University, 253 Industrial Avenue, Guangzhou, Guangdong 510282, P.R. China E-mail: aiminji007@163.com

*Contributed equally

Key words: phosphatidylinositol-4,5-bisphosphate 3-kinase/Akt, apoptosis, cerebral ischemia/reperfusion, brain-derived neurotrophic factor, extracellular signal-regulated kinase 1/2, $\alpha$-lipoic acid of oxidative parameters, including MDA, NO, T-AOC and SOD to their normal state in rat brains following cerebral I/R. Furthermore, the expression of cleaved caspase- 3 markedly decreased and the expression of BDNF, PI3K, p-Akt and p-ERK1/2 significantly increased following administration of LA. On the basis of these findings, it was concluded that LA protected the brain from cerebral I/R damage by attenuation of oxidative stress and caspase-dependent apoptosis. Furthermore, LA exerts its neuroprotective effects potentially through activation of the BDNF-PI3K/Akt-ERK1/2 pathway.

\section{Introduction}

Cerebral ischemic injury is one of the leading causes of human mortality and disability worldwide (1). Restoration of blood flow to the ischemic brain is often used to treat patients in clinical experiments. However, reperfusion itself also has the potential to produce additional injuries in the ischemic brain due to overproduction of reactive oxygen species (ROS). The potential pathological mechanisms of ischemia/reperfusion ( $\mathrm{I} / \mathrm{R})$ injury include glutamate excitotoxicity, calcium overload, nitric oxide (NO) production, oxidative stress, inflammation and apoptosis, which eventually lead to cell death (2). Oxidative stress and apoptosis following cerebral I/R are the two major processes that induce neuronal injury $(3,4)$. For this reason, multifunctional molecules with anti-oxidative and anti-apoptotic properties are ideal neuroprotective agents.

$\alpha$-lipoic acid (LA), an endogenous short-chain fatty acid, is a cofactor for multiple mitochondrial dehydrogenase enzymes, including pyruvate dehydrogenase (5). It is an ROS scavenger, which is able to stimulate the insulin signaling pathway, chelate metal and regenerate endogenous natural antioxidants (6). In previous decades, using several different experimental models of $\mathrm{I} / \mathrm{R}$, studies have demonstrated the protective effects of LA against I/R-induced injury, including myocardial injury (7), peripheral nerve injury (8), testicular injury (9) and retinal injury (10). In addition, it has been reported that LA can activate phosphatidylinositol-4,5-bisphosphate 3-kinase (PI3K)/Akt and extracellular signal-regulated kinase $1 / 2$ (ERK1/2) pathways to induce protection against I/R injury in other organs (11-13). However, 
to the best of our knowledge, few studies were reported to address whether LA has neuroprotective effects against cerebral I/R-induced injury and its potential mechanisms.

In the present study, the neuroprotective effects of LA in rats were investigated with 90 min middle cerebral artery occlusion (MCAO)/24 h reperfusion-induced injury. Furthermore, the hypothesis that the neuroprotective effect of LA is associated with a reduction in oxidative stress and inhibition of apoptosis though activation of brain-derived neurotrophic factor (BDNF), PI3K/Akt and ERK1/2 in rats was assessed. Therefore, the effect of LA on infarct size, neurological deficit score (NDS) and brain water content were investigated. In addition, to examine the mechanisms activated by LA, oxidative parameters, including malondialdehyde (MDA), NO and total antioxidant capacity (T-AOC), superoxide dismutase (SOD) and the expression of cleaved caspase-3, BDNF, PI3K, p-Akt and p-ERK1/2 proteins were measured in rat brains.

\section{Materials and methods}

Animals and drug administration. Adult male Sprague-Dawley rats, 10-12 weeks old, weighing 250-280 g, were purchased from the Animal Center of Southern Medical University (Guangzhou, China). All protocols were approved by the animal care committee of Southern Medical University and undertaken according to the guidelines for the Care and Use of Laboratory Animals of the National Institutes of Health (Bethesda, MD, USA). The rats were kept under constant laboratory conditions $\left(20-25^{\circ} \mathrm{C}, 60 \pm 5 \%\right.$ humidity) and a $12 \mathrm{~h}$ light/dark cycle. They were allowed free access to food and water up until $12 \mathrm{~h}$ prior to surgery at which point only water was available.

LA powder (Shanghai Modern Pharmaceutical Co., Ltd., Shanghai, China) was mixed with sterile saline. Subsequently, $1 \mathrm{M} \mathrm{NaOH}$ was added until the suspension dissolved. The $\mathrm{pH}$ was lowered to 7.4 using hydrochloric acid (14). Rats were administered with LA or saline via subcutaneous injection $2 \mathrm{~h}$ prior to MCAO (15).

Experimental groups. In preliminary experiments, different dosages of LA were administered to determine the optimal dosage. Rats were randomly assigned to the following groups $(n=6)$ : i) Sham group; ii) I/R group, wherein the animals received I/R and a vehicle treatment; iii) LA group, in which the rats received I/R and LA (50 mg/ $\mathrm{kg}$ ); iv) LA group, in which the rats received I/R and LA (70 $\mathrm{mg} / \mathrm{kg}) ; \mathrm{v}) \mathrm{LA}$ group, in which the rats received I/R and LA $(100 \mathrm{mg} / \mathrm{kg})$. Following determining $100 \mathrm{mg} / \mathrm{kg}$ as the optimal dosage, rats were randomly divided into the following groups $(n=9)$ : i) Sham group; ii) I/R group, in which the rats received I/R and a vehicle treatment; iii) LA group, wherein the rats received I/R and LA (100 mg/kg).

Establishment of the MCAO model. Rats fasted overnight, but were allowed free access to water prior to the MCAO procedure. MCAO was induced as described previously (16). Briefly, rats were anesthetized with $10 \%$ chloral hydrate $(3.5 \mathrm{ml} / \mathrm{kg})$ by intraperitoneal injection. The skin and surrounding fur were disinfected with $75 \%$ ethyl alcohol. Following incision to the skin, the left common carotid artery (CCA) was exposed and carefully separated from the vagal nerves. The left external carotid artery (ECA) and the left internal carotid artery (ICA) were carefully isolated and the ECA was ligated. In addition, the CCA was temporally occluded with 3-0 silk thread. The left MCA was occluded for $90 \mathrm{~min}$ with a paraffin-coated nylon filament (17), which was introduced into the ICA for 18-20 mm until resistance was detected. After $90 \mathrm{~min}$ of occlusion, the ECA was permanently occluded. The silk thread occluding the CCA was removed for reperfusion for $24 \mathrm{~h}$. A heating lamp was used to maintain body temperature at $37^{\circ} \mathrm{C}$ during surgery.

Determination of infarct size and neurological function. After $90 \mathrm{~min}$ of ischemia followed by $24 \mathrm{~h}$ of reperfusion animals were anesthetized with $10 \%$ chloral hydrate $(3.5 \mathrm{ml} / \mathrm{kg})$ and decapitated. The brains were quickly removed and frozen at $-20^{\circ} \mathrm{C}$ for $20 \mathrm{~min}$, dissected into $2 \mathrm{~mm}$ coronal slices and immediately incubated in 2\% 2,3,5-triphenyltetrazolium chloride (TTC; Mbchem, Shanghai, China) at $37^{\circ} \mathrm{C}$ for $10 \mathrm{~min}$ as described previously (16). Following TTC staining, the normal brain tissue was dark red, whereas infarcted tissue was unstained. Following TTC staining, the tissues were then fixed in 4\% paraformaldehyde (Mbchem) for $24 \mathrm{~h}$ and scanned with a digital camera (Canon, Inc., Tokyo, Japan). Infarct size was calculated using Image $\mathbf{J}$ software (National Institutes of Health, Bethesda, MD, USA) by an individual blinded to the identity of the experimental groups and the result was expressed as a percentage of infarct area to total brain area.

Neurological deficit evaluations were performed at the end of the experiment by an observer masked to the identity of experimental groups using the following criteria as previously described (16): 0, no neurologic deficit or normal function; 1 , failure to extend right forepaw fully; 2, circling to right; 3 , leaning to right; 4, absence of spontaneous motor activity. Therefore, a higher score was associated with poorer neurological function.

Measurement of brain water content. Brain water content was measured as described previously (18). Briefly, after $24 \mathrm{~h}$ of reperfusion animals were anesthetized with $10 \%$ chloral hydrate $(3.5 \mathrm{ml} / \mathrm{kg})$ and decapitated. The brains were rinsed with saline and separated into ischemic and non-ischemic hemispheres, then immediately weighed to gain the wet weight (WW). The brains were placed in an oven at $100^{\circ} \mathrm{C}$ for $24 \mathrm{~h}$ and weighed to obtain the dry weight (DW). The brain water content (\%) was measured using the following formula: (WW - DW) / WW x 100\%.

Measurement of the oxidative parameters MDA, NO, T-AOC and $S O D$. After $24 \mathrm{~h}$ of reperfusion, animals were anesthetized with $10 \%$ chloral hydrate $(3.5 \mathrm{ml} / \mathrm{kg})$ and decapitated. The rat brains were quickly removed and detached, then rinsed with cold saline. The brains were then homogenized in cold saline $(1: 10, \mathrm{wt} / \mathrm{vol})$ and centrifuged at 3,000 $\mathrm{x}$ g for $10 \mathrm{~min}$ to prepare tissue homogenate. The levels of MDA, NO and the activities of T-AOC and SOD were determined using commercially available assay kits (Nanjing Jiancheng Bioengineering Institute, Nanjing, China) according to the manufacturer's instructions. 
A

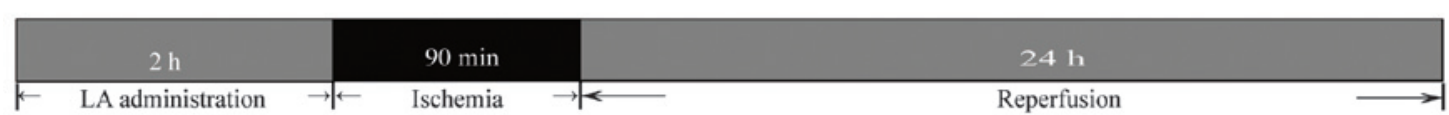

B
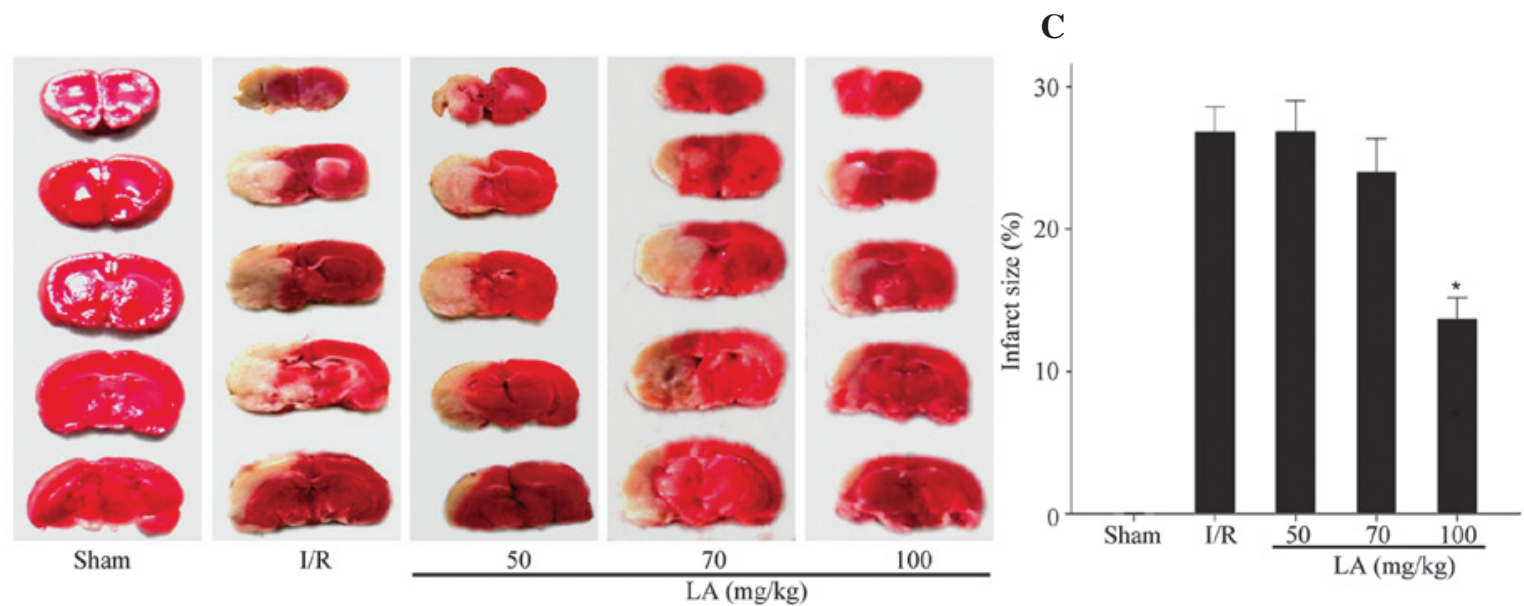

Figure 1. Effects of LA therapy on focal cerebral I/R injury. (A) Experimental procedure. (B) Representative 2,3,5-triphenyltetrazolium chloride staining of cerebral slices, the white-colored areas represent the infarct areas in these sections and the red-colored areas represent non-infarcted areas. (C) Quantification of the infarct size. Rats, with the exception of those in the sham group, underwent transient ischemia by middle cerebral artery occlusion for $90 \mathrm{~min}$ followed by reperfusion for $24 \mathrm{~h}$. Data are presented as the mean \pm standard deviation ( $\mathrm{n}=6$; * $\mathrm{P}<0.05$, LA groups vs. I/R group). LA, $\alpha$-lipoic acid; I/R, ischemia/reperfusion.

Western blotting. Animals were anesthetized with $10 \%$ chloral hydrate $(3.5 \mathrm{ml} / \mathrm{kg})$ and decapitated $24 \mathrm{~h}$ after reperfusion. The rat brains were quickly removed and detached, then rinsed with cold saline. Tissue samples were lysed in ice-cold RIPA buffer (Nanjing Keygen Biotech, Co., Ltd., Nanjing, China) containing $1 \%$ phenylmethylsulfonyl fluoride, $1 \%$ phosphatase inhibitors and $0.1 \%$ protease inhibitor with a glass homogenizer on ice, repeated for $5 \mathrm{~min}$ and incubated on ice for $10 \mathrm{~min}$, then centrifuged at $13,000 \mathrm{x} \mathrm{g}$ for $20 \mathrm{~min}$ at $4^{\circ} \mathrm{C}$. The supernatant was aliquoted and stored at $-80^{\circ} \mathrm{C}$. Protein concentration was measured using the BCA kit (Pierce Biotechnology, Inc., Rockford, IL, USA). The protein (30 $\mu \mathrm{g})$ was then separated on $8-15 \%$ polyacrylamide SDS gels and transferred onto a polyvinylidene difluoride membrane. The membranes were treated with blocking solution (5\% non-fat dry milk or $5 \%$ bovine serum albumin in Tris-buffered saline with Tween ${ }^{\circledR} 20$ and incubated at room temperature for $2 \mathrm{~h}$. Following this, the membranes were incubated with a rabbit anti-rat polyclonal cleaved caspase-3 antibody (1:1,000; Cell Signaling Technology, Inc., Beverly, MA, USA), a rabbit anti-rat polyclonal BDNF antibody (1:1,000; Chemicon, Temecula, CA, USA), a rabbit anti-rat polyclonal PI3K antibody (1:1,000; Cell Signaling Technology, Inc.), a rabbit anti-rat polyclonal p-Akt antibody (1:2,000; Cell Signaling Technology, Inc.) and a rabbit anti-rat polyclonal p-ERK1/2 antibody $(1: 2,000$; Cell Signaling Technology, Inc.) at $4{ }^{\circ} \mathrm{C}$ overnight, followed by incubation with the corresponding goat anti-rabbit secondary antibodies (Wuhan Boster Bio-Engineering Co., Ltd., Wuhan, China) at room temperature for $1 \mathrm{~h}$. GADPH was used as a control to ensure equal protein loading. The blots were visualized with ECL-Plus reagent (Pierce Biotechnology, Inc.) and the exposures were transferred to radiographic films. The radiographic films were scanned by a cannon scanner (Canon,
Inc.) and analyzed using Image $\mathbf{J}$ software (National Institutes of Health).

Statistical analysis. All data were analyzed using SPSS 13.0 software (SPSS, Inc., Chicago, IL, USA) and are expressed as the mean \pm standard deviation. Statistical analysis was performed by one-way analysis of variance and $\mathrm{P}<0.05$ was considered to indicate a statistically significant difference.

\section{Results}

LA attenuates infarct size. The experimental procedure is shown in Fig. 1A. In order to investigate the effect of LA on infarct size, the brain infarct area was analyzed by TCC staining after $24 \mathrm{~h}$ of reperfusion. There was no infarct area in the sham rat brain (Fig. 1B). Pretreatment with $100 \mathrm{mg} / \mathrm{kg}$ LA significantly reduced total infarct size by $51.3 \%$ compared with ischemic rats with vehicle treatment from $26.9 \pm 1.7 \%$ to $13.8 \pm 1.4 \%(\mathrm{P}<0.05)$, while $50 \mathrm{mg} / \mathrm{kg}$ and $70 \mathrm{mg} / \mathrm{kg}$ did not reveal a significant effect $(26.9 \pm 2.1 \%$ and $24.1 \pm 2.3 \%$, respectively; Fig. 1C). Therefore, $100 \mathrm{mg} / \mathrm{kg} \mathrm{LA}$ was selected as the optimum dosage for further investigation.

LA improves neurological function. As shown in Fig. 2A, $24 \mathrm{~h}$ after reperfusion, the neurological score was $3.17 \pm 0.41$ in the I/R group, while this significantly decreased following treatment with LA $(100 \mathrm{mg} / \mathrm{kg})$ to $1.83 \pm 0.41(\mathrm{P}<0.05)$, however, 50 and $70 \mathrm{mg} / \mathrm{kg} \mathrm{LA}$ did not have a significant effect $(3.0 \pm 0.63$ and $2.5 \pm 0.55$, respectively).

LA ameliorates brain edema. Brain water content was quantified using the dry-wet weight method and the result is shown in Fig. 2B. The brain water content in the ischemic area of the 

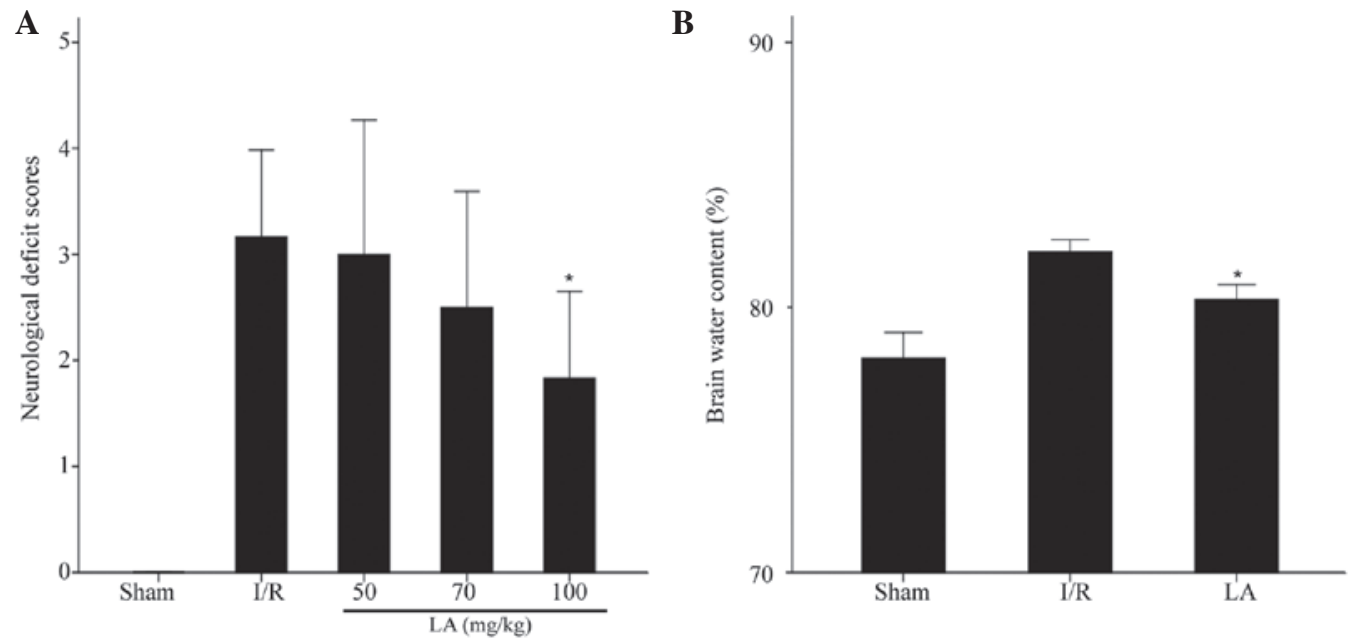

Figure 2. Effects of LA therapy on (A) neurological deficit score and (B) brain water content in rats after 90 min middle cerebral artery occlusion and $24 \mathrm{~h}$ reperfusion. Data are presented as the mean \pm standard deviation $\left(\mathrm{n}=3 ;{ }^{\prime} \mathrm{P}<0.05\right.$, I/R group vs. sham group; ${ }^{*} \mathrm{P}<0.05$, LA group vs. I/R group). LA, $\alpha$-lipoic acid; $\mathrm{I} / \mathrm{R}$, ischemia/reperfusion.

A

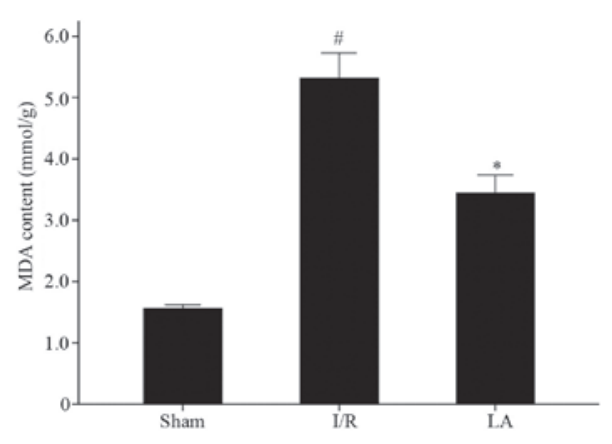

C

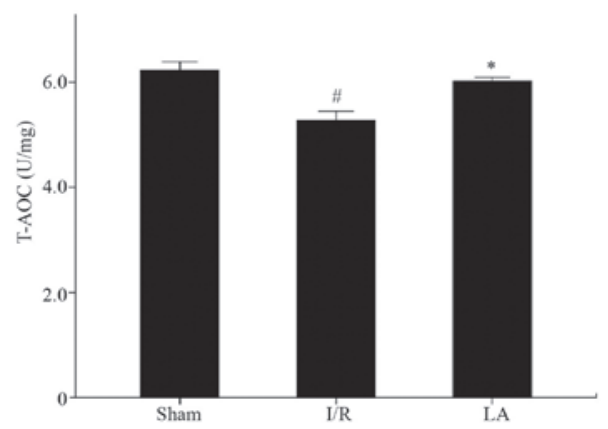

B

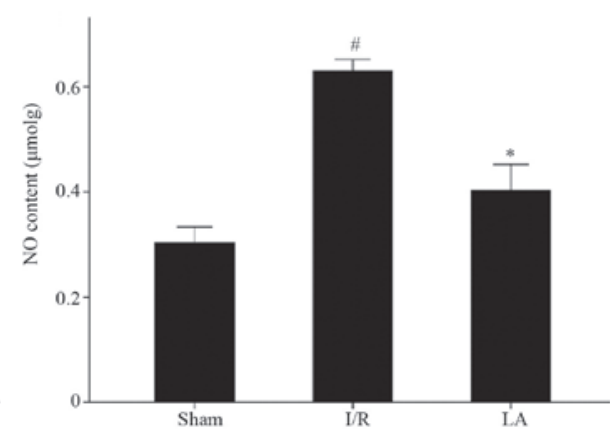

D

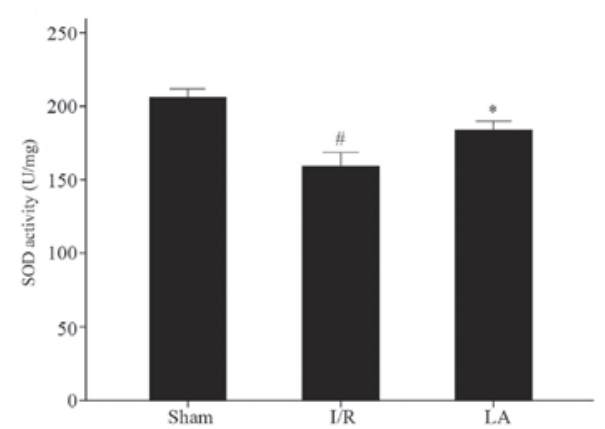

Figure 3. Effects of LA therapy on the oxidative parameters (A) MDA, (B) NO, (C) T-AOC and (D) SOD in ischemic rat brains after 90 min middle cerebral artery occlusion and $24 \mathrm{~h}$ reperfusion. Data are presented as the mean \pm standard deviation $\left(\mathrm{n}=3 ;{ }^{*} \mathrm{P}<0.05, \mathrm{I} / \mathrm{R}\right.$ group vs. sham group; ${ }^{*} \mathrm{P}<0.05, \mathrm{LA}$ group vs. I/R group) LA, $\alpha$-lipoic acid; I/R, ischemia/reperfusion; MDA, malondialdehyde; NO, nitric oxide; T-AOC, total antioxidant capacity; SOD, superoxide dismutase.

I/R group was significantly higher than that of the sham group. Pretreatment of rats with $100 \mathrm{mg} / \mathrm{kg}$ LA significantly reduced brain edema in the rats that underwent $\mathrm{I} / \mathrm{R}(82.1 \pm 0.5 \%$ vs. $80.3 \pm 0.6 \%$; $<0.05)$.

LA improves the oxidative parameters MDA, NO, T-AOC and $S O D$. As shown in Fig. 3, the content of MDA and NO significantly increased in the $\mathrm{I} / \mathrm{R}$ group $(\mathrm{P}<0.05)$ compared with that in the sham group and decreased in the LA-pretreated group
$(\mathrm{P}<0.05)$. The activities of T-AOC and SOD were significantly higher in the LA-treated group $(\mathrm{P}<0.05)$ than in the I/R group.

LA suppresses caspase-independent apoptosis. Cleaved caspase-3, activated from caspase-3, has been identified as a key mediator of apoptosis and is considered to be one of the final steps in cell apoptosis $(19,20)$. As shown in Fig. 4, LA significantly suppressed the level of cleaved caspase-3 compared with the $\mathrm{I} / \mathrm{R}$ group $(\mathrm{P}<0.05)$. 
A

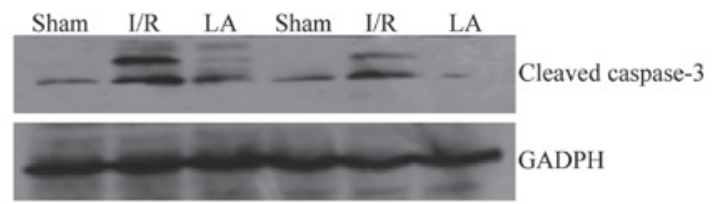

B

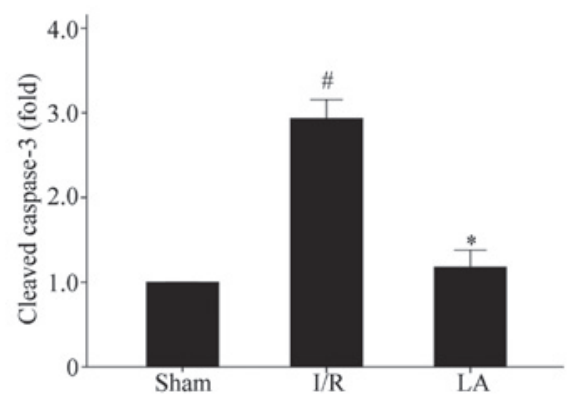

Figure 4. Effects of LA therapy on the protein expression of cleaved caspase-3 in ischemic rat brains after 90 min middle cerebral artery occlusion and $24 \mathrm{~h}$ reperfusion. (A) Representative images of western blotting of cleaved caspase-3. (B) Quantitative data are expressed as the intensity ratio of target protein to GADPH. GADPH was used as a control to ensure equal protein loading. Data are presented as the mean \pm standard deviation $(n=3$; ${ }^{\text {"}} \mathrm{P}<0.05, \mathrm{I} / \mathrm{R}$ group vs. sham group; $\mathrm{P}<0.05$, LA group vs. I/R group). LA, $\alpha$-lipoic acid; $\mathrm{I} / \mathrm{R}$, ischemia/reperfusion.

LA upregulates the expression of BDNF, PI3K, p-Akt and $p$-ERK 1/2. The expression of BDNF, PI3K, p-Akt and p-ERK1/2 were examined using western blotting (Fig. 5A). As shown in Fig. 5B, compared with the I/R group, LA significantly increased the level of BDNF and p-Akt $(\mathrm{P}<0.05)$. The results demonstrated that the levels of PI3K and p-ERK1/2 were significantly downregulated following cerebral I/R, however, pretreatment with LA was able to reverse this effect and increase the expression of these proteins (Fig. 5C-E; P<0.05).

\section{Discussion}

The present study demonstrated that pretreatment with LA has protective effects against neuronal injury caused by cerebral $\mathrm{I} / \mathrm{R}$ in rats. Therefore, as a preventive agent, $\mathrm{LA}$ is beneficial to cerebral I/R-induced injury. This finding is consistent with previous studies on the beneficial effects of LA against cerebral ischemia $(21,22)$.

The results demonstrated that the optimal dose of LA was $100 \mathrm{mg} / \mathrm{kg}$. This was not in accordance with a previous study in which only $5 \mathrm{mg} / \mathrm{kg} \mathrm{LA} \mathrm{had} \mathrm{the} \mathrm{capacity} \mathrm{to} \mathrm{protect} \mathrm{against}$ cerebral I/R-induced injury (22). This difference may be due to the fact that the time period of cerebral $\mathrm{I} / \mathrm{R}$ is longer in the present study ( $30 \mathrm{~min}$ of ischemia followed by $5.5 \mathrm{~h}$ of reperfusion vs. $90 \mathrm{~min}$ of ischemia followed by $24 \mathrm{~h}$ of reperfusion), leading to a more severe neuronal injury. Therefore, a higher dose of $\mathrm{LA}(100 \mathrm{mg} / \mathrm{kg})$ is required to protect against cerebral I/R-induced injury.

Oxidative stress, is the main pathological process of cerebral I/R injury due to the balance between the formation and elimination of ROS, including superoxide, hydrogen peroxide and peroxynitrite (23). The increased concentration of ROS can cause cellular damage and subsequent cell death since ROS may oxidize crucial cellular components, including
A
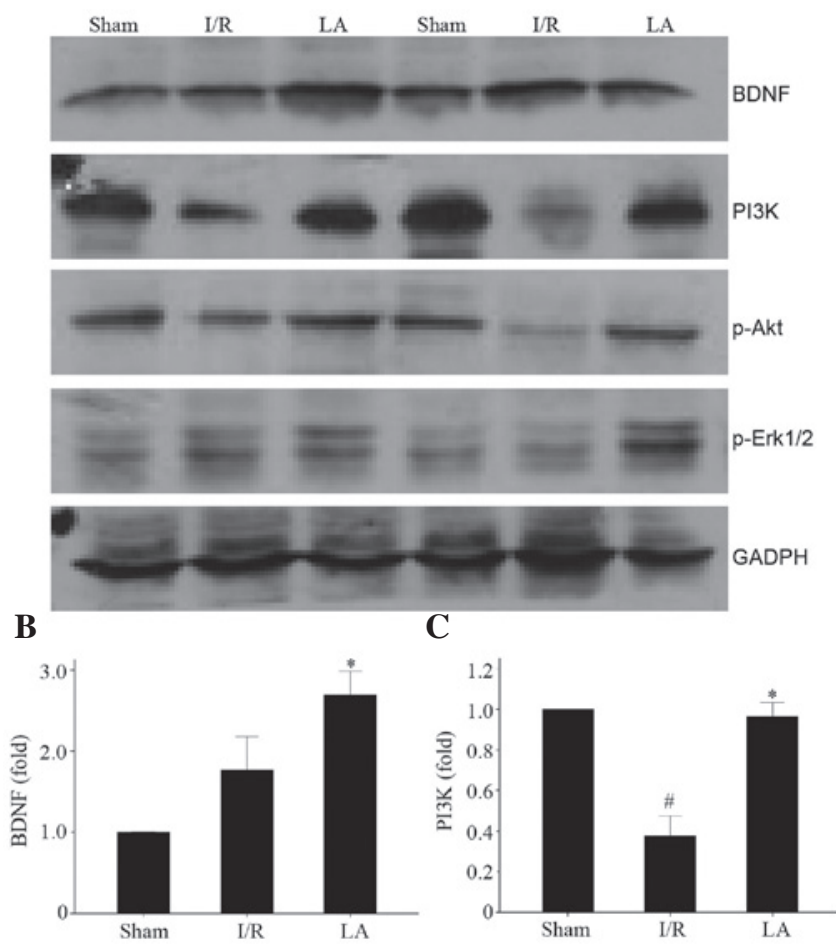

D

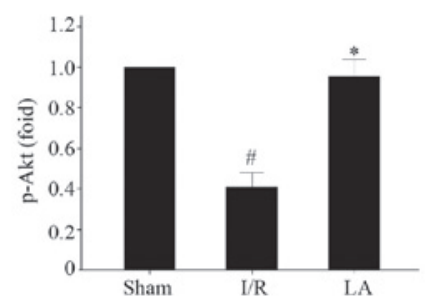

$\mathbf{E}$

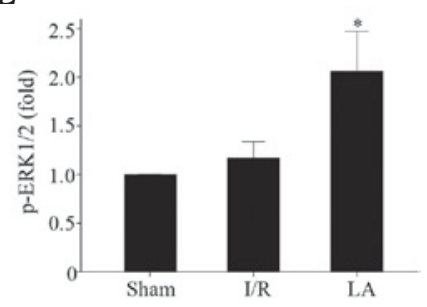

Figure 5. Effects of LA therapy on the protein expression of BDNF, PI3K, p-Akt and p-ERK1/2 in the ischemic brain of rats after 90 min middle cerebral artery occlusion and $24 \mathrm{~h}$ reperfusion. (A) Representative images of western blotting of BDNF, PI3K, p-Akt and p-ERK1/2. (B-E) Quantitative data are expressed as the intensity ratio of target proteins to GADPH. GADPH was used as a control to ensure equal protein loading. Data are presented as the mean \pm standard deviation $\left(\mathrm{n}=3\right.$; ${ }^{*} \mathrm{P}<0.05, \mathrm{I} / \mathrm{R}$ group vs. sham group; ${ }^{*} \mathrm{P}<0.05$, LA group vs. I $/ \mathrm{R}$ group). LA, $\alpha$-lipoic acid; BDNF, brain-derived neurotrophic factor; $\mathrm{I} / \mathrm{R}$, ischemia/reperfusion; PI3K, phosphatidylinositol-4,5-bisphosphate 3-kinase; ERK1/2, extracellular signal-regulated kinase $1 / 2$. p-ERK1/2, phosphoylated extracellular signal-regulated kinase 1/2.

lipids, proteins and DNA (24). The levels of MDA and NO increased indicating that severe oxidative damage was caused by I/R (25). It has been reported that LA has the capability to scavenge MDA and NO within brain tissue (26). In the present study, pretreatment with LA decreased the levels of MDA and NO in rat brains. The endogenous antioxidant enzymes, including SOD and glutathione (GSH) peroxidase and low-molecular weight ROS scavengers, including GSH, are critical in attenuating the injury induced by I/R $(25,27)$. In the present study, LA enhanced the activities of T-AOC and SOD in rat brains. These data provided further evidence that LA could inhibit I/R-induced oxidative stress, which may contribute to the attenuation of $\mathrm{I} / \mathrm{R}$-induced injury.

Apoptosis has a critical pathogenic role in I/R injury. Cleaved caspase- 3 , which is activated from caspase-3, is an important 
molecule involved in apoptosis in I/R injury (28). It is reported that activation of caspase- 3 at the final execution phase of apoptosis leads to DNA fragmentation and cell death (29). It was observed that the level of cleaved caspase-3 markedly increased in the brain following cerebral I/R indicating extensive apoptosis caused by I/R. The present data indicated that pretreatment with LA reversed the increased level of cleaved caspase-3. The result suggested that LA had anti-apoptotic capacity, which was beneficial to cerebral I/R-induced injury.

BDNF is a member of the neurotrophin family and is important in neuronal survival, differentiation, axon growth, dendritic spine development and synaptic plasticity. When a neuron is damaged, BDNF performs a variety of biological effects, including preventing cell death in damaged neurons, improving the pathological state of neurons and promoting the regeneration of damaged neurons (30). In the case of cerebral I/R, BDNF may protect neurons (31-33). BDNF can activate the TrkB receptor, leading to the activation of several intracellular signaling pathways, including PI3K/Akt and RAS/ERK pathways (34). The PI3K/Akt and ERK1/2 signaling pathways are important in regulating cell growth, proliferation and apoptosis (35-37). Numerous studies have reported that the activation of the PI3K/Akt and ERK1/2 pathways are markedly associated with protection from cerebral I/R injury $(38,39)$. Akt, which is a direct downstream target of PI3K when phosphorylated can suppress apoptosis by activation of anti-apoptotic substrates, including B-cell lymphoma 2 family members and inhibition of pro-apoptotic substrates, including cytochrome c (40). When cells suffer ischemic insults, the phosphorylation of Akt may have a protective effect (41). ERK1/2 is a member of the mitogen-activated protein kinase superfamily that can mediate cell proliferation and apoptosis (42). The activation of ERK1/2 and subsequent downstream signaling targets, is important in ROS-mediated cell death (43). In the present study, it was demonstrated that LA significantly increased the level of BDNF. The results revealed that expression of PI3K and p-Akt was significantly downregulated in brains following cerebral I/R, while LA could reverse this situation and increase the levels of PI3K and p-Akt. In addition, the phosphorylation of ERK1/2 increased with administration of LA. The results suggested that LA pretreatment provided protective effects against cerebral I/R-induced injury possibly by promoting the BDNF-PI3K/Akt-ERK1/2 signaling pathway.

In conclusion, the present study demonstrated that LA could afford protection against cerebral I/R-induced injury by attenuation of oxidative stress and caspase-dependent apoptosis. Furthermore, the results suggest that administration of LA induces a neuroprotective effect in association with the activation of the BDNF-PI3K/Akt-ERK1/2 signaling pathway.

\section{Acknowledgements}

This study was supported by the National Science Foundation of China (grant no. 81370449), the Industry, Education and Research of Guangdong Province (grantno.2011B090400015) and the Science and Technology Development project of Guangzhou (grant no. 2010UI-E00531-7).

\section{References}

1. Tu Q, Wang R, Ding B, Zhong W and Cao H: Protective and antioxidant effect of Danshen polysaccharides on cerebral ischemia/reperfusion injury in rats. Int J Biol Macromol 60: 268-271, 2013.

2. Sahota P and Savitz SI: Investigational therapies for ischemic stroke: neuroprotection and neurorecovery. Neurotherapeutics 8: 434-451, 2011.

3. Mattson MP, Duan W, Pedersen WA and Culmsee C: Neurodegenerative disorders and ischemic brain diseases. Apoptosis 6: 69-81, 2001.

4. Zhao ZQ: Oxidative stress-elicited myocardial apoptosis during reperfusion. Curr Opin Pharmacol 4: 159-165, 2004.

5. Perham RN: Swinging arms and swinging domains in multifunctional enzymes: catalytic machines for multistep reactions. Annu Rev Biochem 69: 961-1004, 2000.

6. Smith AR, Shenvi SV, Widlansky M, Suh JH and Hagen TM: Lipoic acid as a potential therapy for chronic diseases associated with oxidative stress. Curr Med Chem 11: 1135-1146, 2004.

7. Wang X, Yu Y, Ji L, Liang X, Zhang T and Hai CX: Alpha-lipoic acid protects against myocardial ischemia/reperfusion injury via multiple target effects. Food Chem Toxicol 49: 2750-2757, 2011.

8. Mitsui Y, Schmelzer JD, Zollman PJ, Mitsui M, Tritschler HJ and Low PA: Alpha-lipoic acid provides neuroprotection from ischemia-reperfusion injury of peripheral nerve. J Neurol Sci 163: $11-16,1999$

9. Ozbal S, Ergur BU, Erbil G, Tekmen I, Bagriyanık A and Cavdar Z: The effects of $\alpha$-lipoic acid against testicular ischemia-reperfusion injury in rats. ScientificWorldJournal 2012: 489248, 2012.

10. Chidlow G, Schmidt KG, Wood JP, Melena J and Osborne NN: Alpha-lipoic acid protects the retina against ischemia-reperfusion. Neuropharmacology 43: 1015-1025, 2002.

11. Deng C, Sun Z, Tong G, et al: $\alpha$-Lipoic acid reduces infarct size and preserves cardiac function in rat myocardial ischemia/reperfusion injury through activation of PI3K/Akt/Nrf2 pathway. PLoS One 8: e58371, 2013.

12. Oh SK, Yun KH, Yoo NJ, et al: Cardioprotective effects of alpha-lipoic acid on myocardial reperfusion injury: suppression of reactive oxygen species generation and activation of mitogen-activated protein kinase. Korean Circ J 39: 359-366, 2009.

13. XieR,LiX,Ling Y,etal:Alpha-lipoic acidpre-and post-treatments provide protection against in vitro ischemia-reperfusion injury in cerebral endothelial cells via Akt/mTOR signaling. Brain Res 1482: 81-90, 2012.

14. Cameron NE, Cotter MA, Horrobin DH and Tritschler HJ: Effects of alpha-lipoic acid on neurovascular function in diabetic rats: interaction with essential fatty acids. Diabetologia 41: 390-399, 1998.

15. Wolz P and Krieglstein J: Neuroprotective effects of alpha-lipoic acid and its enantiomers demonstrated in rodent models of focal cerebral ischemia. Neuropharmacology 35: 369-375, 1996.

16. Longa EZ, Weinstein PR, Carlson S and Cummins R: Reversible middle cerebral artery occlusion without craniectomy in rats. Stroke 20: 84-91, 1989.

17. Zuo XL, Wu P and Ji AM: Nylon filament coated with paraffin for intraluminal permanent middle cerebral artery occlusion in rats. Neurosci Lett 519: 42-46, 2012.

18. Lan R, Xiang J, Wang GH, et al: Xiao-Xu-Ming decoction protects against blood-brain barrier disruption and neurological injury induced by cerebral ischemia and reperfusion in rats. Evid Based Complement Alternat Med 2013: 629782, 2013.

19. Namura S, Zhu J, Fink K, et al: Activation and cleavage of caspase-3 in apoptosis induced by experimental cerebral ischemia. J Neurosci 18: 3659-3668, 1998

20. D'Amelio M, Cavallucci V and Cecconi F: Neuronal caspase-3 signaling: not only cell death. Cell Death Differ 17: 1104-1114, 2010.

21. Clark WM, Rinker LG, Lessov NS, Lowery SL and Cipolla MJ: Efficacy of antioxidant therapies in transient focal ischemia in mice. Stroke 32: 1000-1004, 2001.

22. Connell BJ, Saleh M, Khan BV and Saleh TM: Lipoic acid protects against reperfusion injury in the early stages of cerebral ischemia. Brain Res 1375: 128-136, 2011.

23. Yabuki Y and Fukunaga K: Oral administration of glutathione improves memory deficits following transient brain ischemia by reducing brain oxidative stress. Neuroscience 250: 394-407, 2013. 
24. Zhou XQ, Zeng XN, Kong H and Sun XL: Neuroprotective effects of berberine on stroke models in vitro and in vivo. Neurosci Lett 447: 31-36, 2008.

25. Wang PR, Wang JS, Zhang C, Song XF, Tian N and Kong LY: Huang-Lian-Jie-Du-Decotion induced protective autophagy against the injury of cerebral ischemia/reperfusion via MAPK-mTOR signaling pathway. J Ethnopharmacol 149: 270-280, 2013

26. Packer L, Tritschler HJ and Wessel K: Neuroprotection by the metabolic antioxidant alpha-lipoic acid. Free Radic Biol Med 22: 359-378, 1997.

27. Yang G, Chan PH, Chen J, et al: Human copper-zinc superoxide dismutase transgenic mice are highly resistant to reperfusion injury after focal cerebral ischemia. Stroke 25: 165-170, 1994.

28. Haylor JL, Harris KP, Nicholson ML, Waller HL, Huang Q and Yang B: Atorvastatin improving renal ischemia reperfusion injury via direct inhibition of active caspase-3 in rats. Exp Biol Med (Maywood) 236: 755-763, 2011.

29. Thornberry NA and Lazebnik Y: Caspases: enemies within. Science 281: 1312-1316, 1998.

30. Binder DK and Scharfman HE: Brain-derived neurotrophic factor. Growth Factors 22: 123-131, 2004.

31. Ferrer I, Krupinski J, Goutan E, Marti E, Ambrosio S and Arenas E: Brain-derived neurotrophic factor reduces cortical cell death by ischemia after middle cerebral artery occlusion in the rat. Acta Neuropathol 101: 229-238, 2001.

32. Muller HD, Hanumanthiah KM, Diederich K, Schwab S, Schabitz WR and Sommer C: Brain-derived neurotrophic factor but not forced arm use improves long-term outcome after photothrombotic stroke and transiently upregulates binding densities of excitatory glutamate receptors in the rat brain. Stroke 39: 1012-1021, 2008

33. Ploughman M, Windle V, MacLellan CL, White N, Doré JJ, and Corbett D: Brain-derived neurotrophic factor contributes to recovery of skilled reaching after focal ischemia in rats. Stroke 40: 1490-1495, 2009.
34. Patapoutian A and Reichardt LF: Trk receptors: mediators of neurotrophin action. Curr Opin Neurobiol 11: 272-280, 2001.

35. Burgering BM and Coffer PJ: Protein kinase B (c-Akt) in phosphatidylinositol-3-OH kinase signal transduction. Nature 376 599-602, 1995.

36. Franke TF, Yang SI, Chan TO, et al: The protein kinase encoded by the Akt proto-oncogene is a target of the PDGF-activated phosphatidylinositol 3-kinase. Cell 81: 727-736, 1995.

37. Xia Z, Dickens M, Raingeaud J, Davis RJ and Greenberg ME: Opposing effects of ERK and JNK-p38 MAP kinases on apoptosis. Science 270: 1326-1331, 1995.

38. Arslan F, Lai RC, Smeets MB, et al: Mesenchymal stem cell-derived exosomes increase ATP levels, decrease oxidative stress and activate PI3K/Akt pathway to enhance myocardial viability and prevent adverse remodeling after myocardial ischemia/reperfusion injury. Stem Cell Res 10: 301-312, 2013.

39. Zhou L and Miller CA: Mitogen-activated protein kinase signaling, oxygen sensors and hypoxic induction of neurogenesis. Neurodegener Dis 3: 50-55, 2006.

40. Franke TF, Hornik CP, Segev L, Shostak GA and Sugimoto C: PI3K/Akt and apoptosis: size matters. Oncogene 22: 8983-8998, 2003.

41. Liu H, Liu X, Wei X, et al: Losartan, an angiotensin II type 1 receptor blocker, ameliorates cerebral ischemia-reperfusion injury via PI3K/Akt-mediated eNOS phosphorylation. Brain Res Bull 89: 65-70, 2012.

42. Mebratu Y and Tesfaigzi Y: How ERK1/2 activation controls cell proliferation and cell death: Is subcellular localization the answer? Cell Cycle 8: 1168-1175, 2009.

43. Dong J, Ramachandiran S, Tikoo K, Jia Z, Lau SS and Monks TJ: EGFR-independent activation of p38 MAPK and EGFR-dependent activation of ERK1/2 are required for ROS-induced renal cell death. Am J Physiol Renal Physiol 287: F1049-F1058, 2004. 